\title{
Peri-Aneurysmal Brain Edema in Native and Treated Aneurysms: The Role of Thrombosis
}

\author{
Valeria Onofrj, $\mathrm{MD}^{1}$, Donatella Tampieri, $\mathrm{MD}^{2}$, Alessandro Cianfoni, $\mathrm{MD}, \mathrm{PhD}^{1,3}$, Elisa Ventura, $\mathrm{MD}^{1}$ \\ ${ }^{1}$ Department of Neuroradiology, Neurocenter of Southern Switzerland, Ospedale Regionale di Lugano, Lugano, Switzerland \\ 2Department of Radiology, Queen's University at Kingston, Kingston, ON, Canada \\ ${ }^{3}$ Department of Neuroradiology, Inselspital, Bern University Hospital, University of Bern, Bern, Switzerland
}

Cerebral peri-aneurysmal edema (PE) is typically associated with giant partially-thrombosed aneurysms and less frequently with smaller aneurysms treated with endovascular embolization. An understanding of the pathophysiologic mechanism of PE is still limited. We report 3 cases of cerebral aneurysms associated with PE. We describe 2 cases of giant partially thrombosed aneurysms surrounded by vasogenic edema with apposition of an intramural and juxtamural thrombus. Our third case is a smaller aneurysm inciting vasogenic edema several years after coil embolization. Vessel-wall magnetic resonance imaging (MRI) showed avid wall enhancement and an enhancing thrombus embedded within the coils, reflecting inflammation of the aneurysm wall and proliferation of the vasa vasorum. Thrombosis within the aneurysmal sac and walls, both in native and treated aneurysms, may promote inflammatory changes and sustain the occurrence of PE. Vessel-wall MRI has a potential role in the evaluation process of this subgroup of aneurysms.

Key Words: Cerebral aneurysms; Thrombosis; Perianeurysmal edema; Inflammation; Magnetic resonance vessel-wall imaging

\section{INTRODUCTION}

Cerebral peri-aneurysmal edema (PE) is a rare phenomenon with a poorly understood pathophysiologic mechanism. It has been primarily described in association with partially thrombosed giant aneurysms, presenting with an onset of acute symptoms, such as seizure, headache, and neurological deficits, ${ }^{1}$ and has generally been explained as a result of mass effect due to acute swelling of the aneurysm following thrombosis. ${ }^{2}$

Less frequently PE has been reported in smaller aneurysms after endovascular coil embolization, suggesting a "foreign body" inflammatory response, possibly explaining vasogenic edema in the absence of a significant mass effect. ${ }^{3,4}$

Magnetic resonance vessel-wall imaging (MR VWI) has recently demonstrated that PE occurs only in association with aneurysm wall enhancement, even in small aneurysms not exerting significant mass effect on the adjacent brain parenchyma ${ }^{5}$ which possibly reflects a non-specific inflammatory process with the proliferation of vasa vasorum in the vessel wall. ${ }^{6}$ However, the triggers of this inflammatory response have yet to be

\section{Correspondence to:} Valeria Onofrj, MD Department of Neuroradiology, Neurocenter of Southern Switzerland, Ospedale Civico di Lugano, via Tesserete 46, 6900, Lugano, Switzerland Tel: +41792938595

Fax: +41792938595

E-mail: valeriaonofrj@gmail.com

Received: July 7, 2020

Revised: October 30, 2020

Accepted: November 12, 2020
Copyright $\odot 2021$ Korean Society of Interventional Neuroradiology This is an Open Access article distributed under the terms of the Creative Commons Attribution Non-Commercial License (http://creativecommons.org/licenses/by-nc/4.0) which permits unrestricted non-commercial use, distribution, and reproduction in any medium, provided the original work is properly cited.

pISSN 2093-9043 eISSN 2233-6273 
established.

We report 3 cases of PE, 2 associated with partially thrombosed giant aneurysms, and 1 with a smaller aneurysm inciting vasogenic edema several years after endovascular coil embolization. We identify the imaging findings that our 3 cases have in common and discuss the possible mechanisms triggering and sustaining PE.

\section{CASE REPORT}

This case series includes radiographic and clinical information of 3 patients. The data were analyzed retrospectively after our institutional review board waived the approval for the present case series.

We selected 3 cases of partially thrombosed aneurysms with PE and reviewed the patients' charts and imaging studies. Patients were included in the study regardless of the aneurysm size and morphology. Exclusion criteria were as follows: previous aneurysm rupture, presence of other brain expansile lesions possibly associated to vasogenic edema, and a known history of underlying vasculopathy.

\section{Case 1}

A 55-year-old male presented with generalized seizures. There was no past medical history of high blood pressure (HBP), diabetes (DM), no known vasculopathy, coagulopathy, heart or blood vessel disease, and no history of smoking. No family history of ruptured/unruptured aneurysm was reported. Admission head computed tomography (CT), followed by magnetic resonance imaging (MRI), showed a $3.5 \mathrm{~cm}$ giant aneurysm of the left middle cerebral artery (MCA), with a juxtamural thrombus and narrowing of the patent lumen surrounded by vasogenic edema in the adjacent temporal lobe white matter (Fig. 1A, B). Diffusion-weighted imaging ruled out acute ischemic lesions.

Catheter digital subtraction angiography (DSA) further characterized the patent aneurysm lumen, narrowed by the juxtamural thrombus at the inferior aspect of the aneurysm sac (Fig. 1C).

The patient underwent steroid treatment with Dexamethasone ( $0.1 \mathrm{mg} / \mathrm{kg} /$ day per os) resulting in significant interval improvement of the PE at the one-month follow-up CT scan (Fig. 1D). Treatment was postponed to 1 month after the initial presentation in consideration of previous studies showing a continuously growing and dramatic increase in vasogenic edema of giant, partially thrombosed aneurysms following selective coiling or even after aneurysm clipping. This known tendency to rapid growth, which happened to be fatal in some cases, makes giant partially thrombosed aneurysms candidates for treatment with parent vessel occlusion. ${ }^{2,7-9}$ However, in our case, parent vessel occlusion was not feasible; therefore, we decided to first decrease vasogenic edema with steroids and treat the patient with a flow-diverter implant.

A follow-up DSA showed a mildly larger patent aneurysm (Fig. 1E) due to remodeling of the thrombosed juxta-mural component with consequent mild enlargement of the aneurysm lumen. The aneurysm was subsequently treated with an implant of a flow-diverter and loose intrasaccular coiling with proximal residual lumen on early post-treatment DSA (Fig. 1F). The post-treatment course was uneventful; a 3-month follow-up CT-scan showed almost complete resolution of the PE (not shown).

\section{Case 2}

A 23-year-old female presented with acute onset of headache and right hemiparesis, after a 2-week history of fluctuating weakness of the right upper and lower limbs. There was no past medical history of HBP, DM, heart or blood vessel disease, no known history of coagulopathy, vasculopathy, and no history of smoking. No family history of ruptured/ unruptured aneurysm was reported.

Head-CT and CT-angiography (Fig. 2A, B) showed a giant aneurysm, $2.7 \mathrm{~cm}$ in maximum diameter, with extensive intramural and juxtamural thrombus, along the course of the P3 tract of the left posterior cerebral artery. The DSA confirmed a dissecting aneurysm of the left P3 segment with a small (6 mm) perfused lumen (Fig. 2C).

On a MR heterogeneous signal, an "onion-skin" pattern was visible in the aneurysmal clot, reflecting blood products of different ages, and a significant amount of vasogenic edema was present in the left cerebral peduncle, thalamus, and posterior limb of the internal capsule (Fig. 2D, E). Subtle contrast-enhancement was detected after intravenous administration of gadolinium along the aneurysm wall (Fig. 2F).

Endovascular treatment with a flow-diverter implant was performed. The post-procedure course was uneventful. Follow-up MR and 1-month post-procedure showed marked shrinkage of the thrombus, with a resolution of the PE (Fig. 2G, H). Follow-up DSA showed complete occlusion of the aneurysmal sac (Fig. 21). 


\section{Case 3}

A 79-year-old female was admitted after a first seizure. She had a past medical history 12 years prior of an unruptured left MCA aneurysm treated with endovascular treatment with bare platinum coils. The patient had no past medical history of HBP, DM, vasculopathy or coagulopathy, and no history of smoking. No family history of ruptured/unruptured aneurysm was reported. MR and magnetic resonance angiography images before treatment showed a $10 \mathrm{~mm}$ left MCA aneurysm with minimal surrounding PE (Fig. 3A, B).
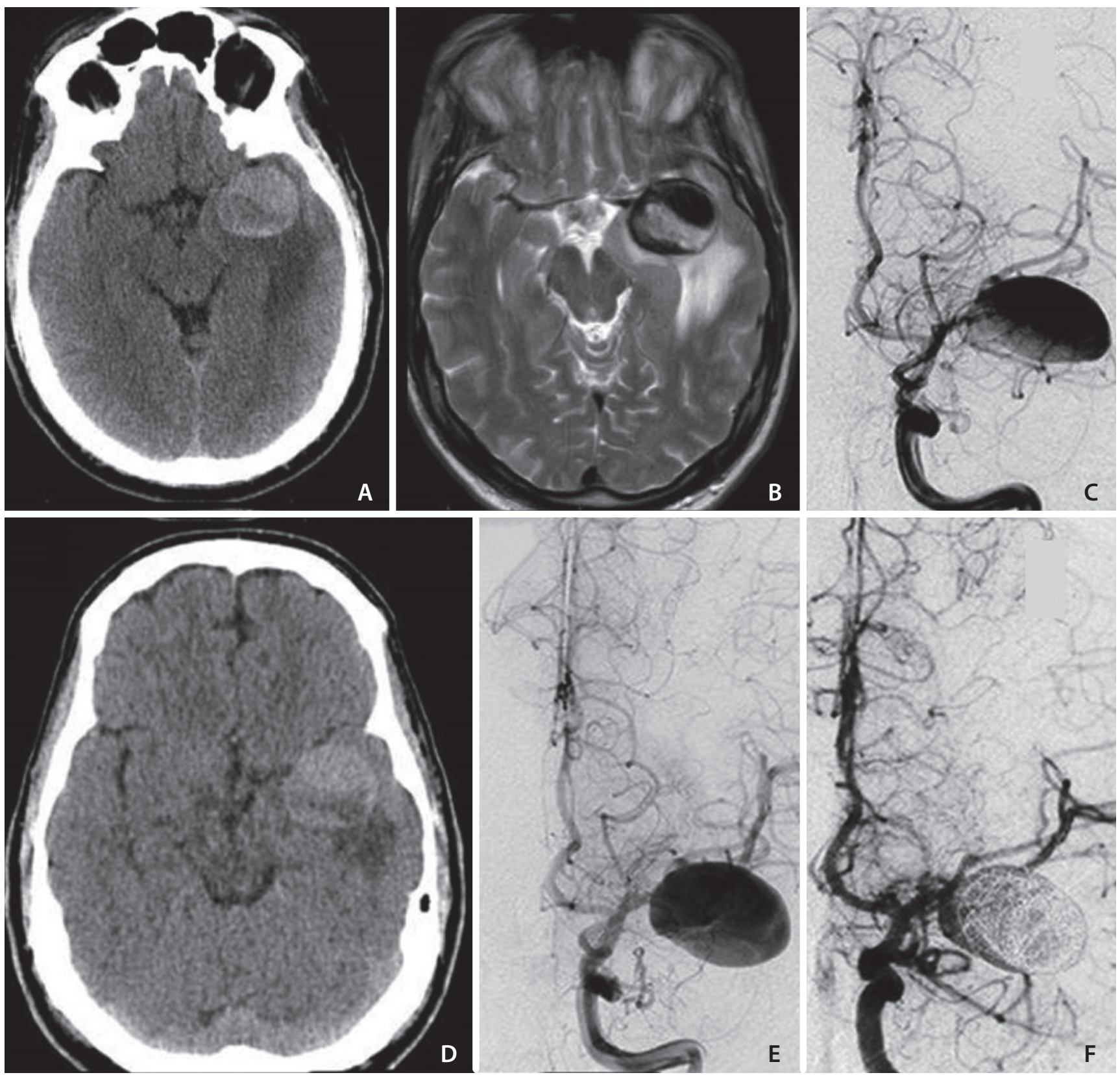

Fig. 1. Case 1. A 55 years-old male presenting with generalized seizures. On admission, non contrast computed tomography (NCCT) suggests the presence of a giant partially-thrombosed aneurysm of the left middle cerebral artery (MCA) inciting vasogenic edema in the surrounding brain parenchyma (A). A significant amount of peri-aneurysmal edema (PE) is confirmed on a T2-weighted axial image (B) in the white matter of the left temporal lobe resulting in mass effect on the ipsilateral cerebral peduncle. The aneurysm lumen shows signal flow-void in keeping with patency of the lumen. Catheter digital subtraction angiography (DSA) demonstrates the left MCA aneurysm (C) and its mild increase in size in a follow-up study (E) regardless of the interval decrease of PE on NCCT (D). Early post-treatment DSA after flow-diverter implant and loose intrasaccular coiling shows residual blood-flow in the proximal lumen $(\mathbf{F})$. 

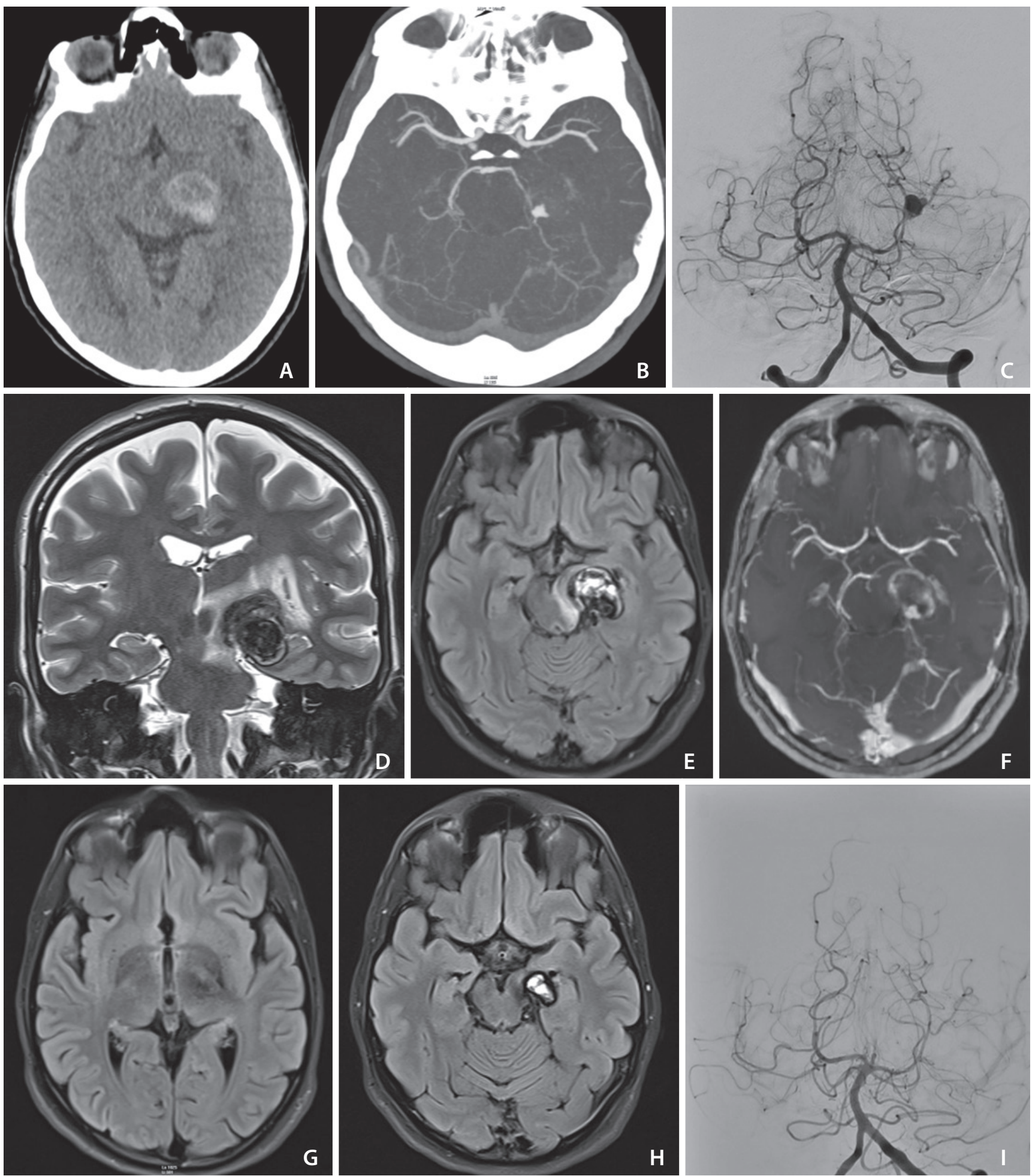

Fig. 2. Case 2. A 23 years-old female with right hemiparesis. Head non contrast computed tomography (NCCT) (A) and CT angiography (CTA) (B) after intravenous (i.v.) administration of iodinated contrast medium show a partially-thrombosed giant aneurysm of the left posterior cerebral artery (P3 tract) with a small patent lumen (B) confirmed at catheter digital subtraction angiography (DSA) (C). Magnetic resonance (MR) reveals a significant amount of PE in the left cerebral peduncle and thalamo-capsular region (D) and demonstrates the heterogeneous signal intensity of the intraluminal clot suggesting repetitive intra-aneurysmal hemorrhage (E). Subtle contrast-enhancement is noted along the aneurysmal walls after i.v. administration of gadolinium (F). A 1-month follow-up MR study after endovascular treatment reveals the complete resolution of peri-aneurysmal edema and visible shrinkage of the thrombus $(\mathbf{G}, \mathbf{H})$. No signs of recanalization of the treated aneurysm are observed on post-treatment DSA (I). 
Sagittal T1-weighted images suggested the presence of a hyperintense intraluminal clot within the aneurysm (Fig. 3C). On neuroimaging performed 2 days after endovascular embolization with coils, no signs of residual aneurysmal sac perfusion were observed on 3D-time of flight (TOF) images (Fig. 3D), and the amount of PE was stable. The coiled aneurysm and the surrounding vasogenic edema remained unchanged over several imaging follow-ups. However,
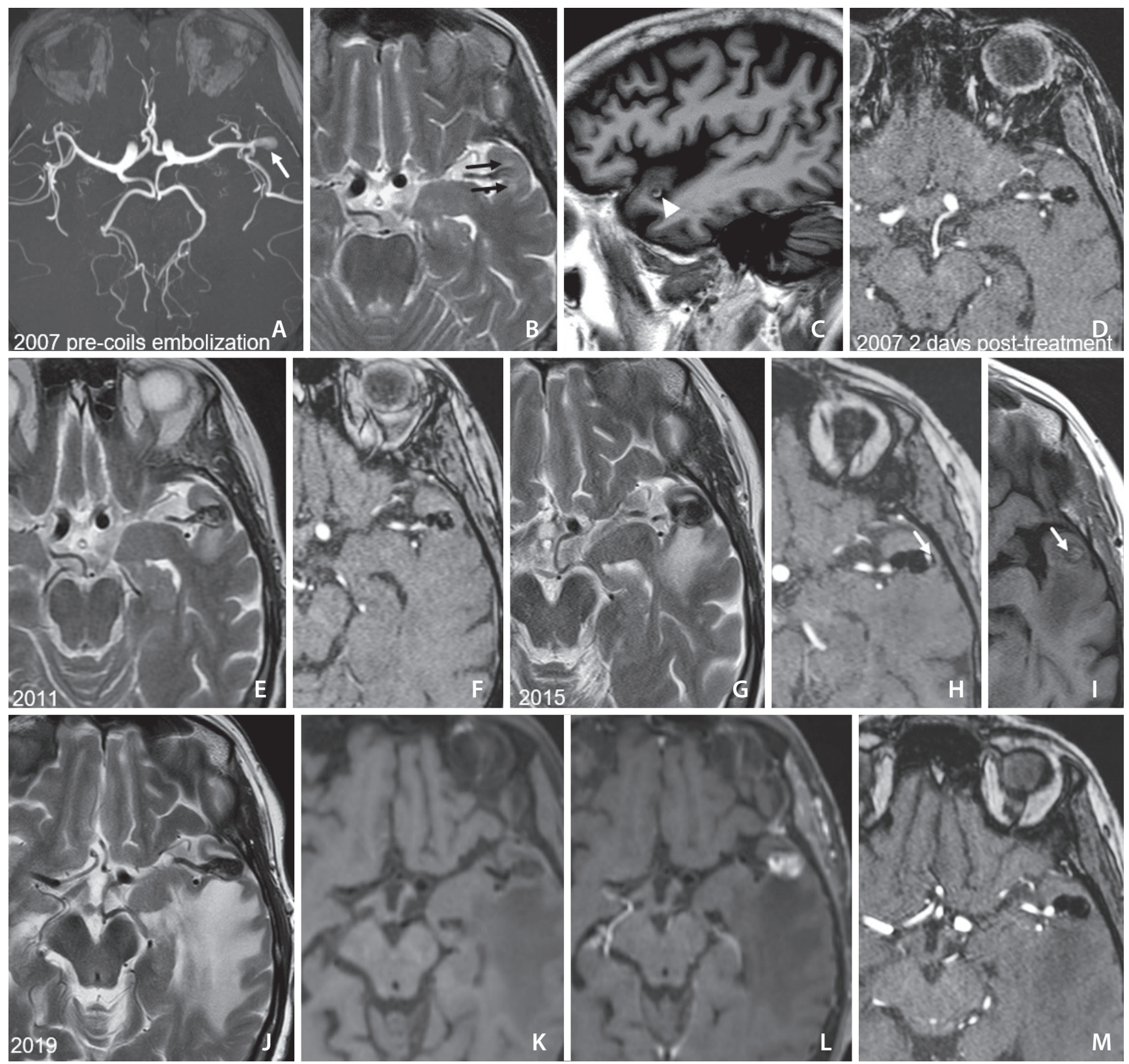

Fig. 3. Case 3. A 79 years old female with a sudden episode of seizure. Magnetic resonance imaging and magnetic resonance angiography (MRA) before endovascular treatment (2007) show a 10 mm left middle cerebral artery (MCA) aneurysm (white arrow in A) with minimal peri-aneurysmal edema (PE) surrounding the aneurysmal sac (black arrows in B) and a small T1 hyperintense intra-luminal clot (white arrowhead in C). MRA 2 days after coils embolization shows no intraluminal filling of the treated aneurysm (D). Follow-up MR studies respectively 4 (2011), 8 (2015) and 12 (2019) years after initial treatment reveal a progressive and significant increase in PE $(\mathbf{E}, \mathbf{G}, \mathbf{J})$ despite no interval recanalization of the left MCA aneurysm on 3D-time of flight (TOF) images ( $\mathbf{F}, \mathbf{H}, \mathbf{M})$. A small T1 hyperintense spot is observed within the aneurysm in the 8-year post-treatment study on TOF and T1W images respectively (white arrows in $\mathbf{H}$ and $\mathbf{I}$ ), indicating new apposition of the intraluminal clot. Axial T1-space images before and after i.v administration of gadolinium $(\mathbf{K}, \mathbf{L})$ demonstrate vivid contrast-enhancement of the aneurysmal walls and sac suggestive of ongoing inflammatory changes. 
MRI performed 4 years after the initial treatment showed increased PE without evidence of aneurysm recanalization (Fig. 3E, F). On a follow-up MRI, 8 years after the initial treatment, the aneurysm demonstrated a slight enlargement in size. The surrounding vasogenic edema was also mildly increased from prior studies, despite the absence of intraluminal flow on angiographic 3D-TOF images (Fig. 3G, H). In addition, a new focus on the $\mathrm{T} 1$ hyperintense signal was observed along the aneurysm fundus on both TOF and T1-weighted images, reflecting new intraluminal clot formation (Fig. 3G-I). Because of the absence of aneurysm recanalization and the evidence of new clot formation, we interpreted the subtle enlargement of the aneurysm as a result of aneurysm-wall remodeling. The coil mass within the aneurysm lumen did not show any significant change in morphology compared to previous studies.

MRI on admission, almost 12 years after endovascular treatment, showed markedly increased vasogenic edema extending to the white matter of the left temporal and parietal lobes, insula, and internal capsule with mild mass effect on the temporal horn of the left ventricle. The left temporal gyri appeared swollen.

Perianeurysmal vasogenic edema has been rarely reported in association with small aneurysms in association with vessel-wall enhancement; ${ }^{3,4}$ therefore, we decided to complete the MRI study with "black-blood" T1-SPACE (Sampling Perfection with Application optimized Contrasts using different flip angle Evolution) pre- and post-contrast sequences with isotropic $2 \times 2 \times 2 \mathrm{~mm}^{3}$ size. Vivid intraluminal and aneurysmal wall enhancement was observed on the post-contrast T1-SPACE sequence, with no signs of aneurysm recanalization on 3D-TOF images, in keeping with the presence of ongoing inflammatory phenomena within the coils-clot complex (Fig. 3J-M). No additional treatment was prescribed at this time, as the patient was already treated with corticosteroids for a long-standing medical history of polymyalgia rheumatica. A follow-up MRI study performed after 1 year from the onset of seizures demonstrated stable intraluminal and peripheral aneurysm wall enhancement and unchanged PE, suggesting persistent active inflammation of the intraluminal thrombus.

\section{DISCUSSION}

PE is a rare phenomenon mostly observed in association with giant intracranial aneurysms. Intuitively, as with brain tumors or intracerebral hemorrhage, large aneurysms may exert a mass effect on brain parenchyma resulting in venous system compression and vasogenic edema, with the potential additional mechanism of the "water hammer effect" due to transmitted pulsations on adjacent brain structures. ${ }^{1,2}$ Dengler et al. ${ }^{10}$ reported in their retrospective study that partial thrombosis of intracranial aneurysms may represent an even more significant risk factor for the occurrence of PE. In fact, only one-third of giant aneurysms seem to present with $P E$, whereas almost all giant aneurysms with PE appear to be partially thrombosed. ${ }^{8}$

Unlike saccular aneurysms, partially thrombosed aneurysms present typically with progressive growth and perianeurysmal edema and only seldom with rupture and subarachnoid hemorrhage. ${ }^{9}$ According to Krings et al. ${ }^{9,11}$, partially thrombosed giant aneurysms represent, from a histopathological point of view, a specific subgroup of unstable, potentially-growing intracranial aneurysms. Repeated intramural or juxtamural hemorrhage (either within the aneurysm wall or between the aneurysm wall and the old thrombus at the periphery of the lumen) may explain their tendency to grow even without evidence of lumen recanalization. lihara et al. ${ }^{8}$ reported a case of a giant vertebral artery aneurysm continuing to grow despite successful endovascular embolization with coils and a lack of angiographically proven aneurysmal intrasaccular flow. Magnetic resonance imaging revealed a marked enhancement around the packed coils close to the neck of the aneurysm. Histological examination showed the presence of inflammatory cells and neovascularization of a partially organized thrombus around the packed coils in the aneurysm and marked development of vasa vasorum along the aneurysm neck.

PE has been rarely reported in smaller, non-giant intracranial aneurysms, following endovascular embolization with coils. An increased number of PE cases became evident after the introduction of modified "active" coils, including polyglycolic acid/polylactic acid coils (Matrix coils) and coils with an expandable gel coating to achieve a tighter packing (HydroCoil). ${ }^{3,4,12}$ MR VWI, an increasingly useful tool for the assessment of aneurysm wall pathology, has demonstrated the occurrence of aneurysm wall-enhancement in all cases of peri-aneurysmal edema presenting without the evidence of significant mass effect. ${ }^{6,13}$ In line with recent histopathological studies, some authors have attributed wall enhancement occurring with perianeurysmal edema to an inflammatory 
response of the aneurysm wall with neoangiogenesis of the vasa-vasorum triggered by a "foreign body" reaction to the coils within the aneurysm lumen. ${ }^{3,4,12}$ However, only a limited number of aneurysms treated with coils present with vasogenic edema, thus it seems plausible to hypothesize that other factors are needed to trigger and sustain an inflammatory reaction.

The pathophysiology of thrombosis and inflammation are known to be interlinked by different factors. ${ }^{14}$ In light of this, thrombosis may play a central role in triggering and boosting adventitial inflammation, promoting neoangiogenesis, and leading to an increased supply of inflammatory cells to the artery wall. This inflammatory response may eventually create a vicious circle with recruitment of activated leukocytes in cerebral venules surrounding the aneurysm and release of different factors (ie, oxygen radicals, matrix metalloproteinase) that increase the permeability of the blood-brain-barrier and cause vasogenic edema. ${ }^{15}$

We reported 2 cases of giant partially thrombosed aneurysms with juxtamural and intramural thrombus, 1 of which, a giant dissecting aneurysm, showed wall enhancement, in keeping with aneurysm wall inflammation. The most relevant findings of our third case-a treated, non-recanalized $10 \mathrm{~mm}$ aneurysm — were the evidence of active enhancing thrombus embedded with the embolizing coils within the aneurysm lumen, the avid enhancement and inflammation of the aneurysmal walls, and the progressive and extensive increase in surrounding vasogenic edema in the follow-up MR studies. Of notice, vasogenic edema was present in a very small amount, even before aneurysm coiling, thus excluding the hypothesis of a "foreign body" reaction to the coils. We also observed that the intraluminal thrombosis was likely present even before the endovascular treatment, with new thrombotic apposition along the aneurysm wall seen over the course of several years despite stable aneurysm occlusion. These findings may imply that constant formation of thrombus within the aneurysm lumen may trigger the inflammatory response of the adventitia eventually causing vasogenic edema.

Our case series highlights that aneurysm wall enhancement in association with endoluminal and transmural thrombosis may serve as an imaging biomarker for aneurysm wall inflammation, revealing possible aneurysmal instability and a higher risk of rupture. Nonetheless, current evidence of MR VWI in a clinical role is limited. ${ }^{6,13}$ Lack of longitudinal studies, heterogeneity of MR protocols, imaging pitfalls related to pulsation artifacts, and controversies on the definition of "wall-enhancement" warrant a critical appraisal. ${ }^{6,13,16}$ Prospective studies are needed to fully comprehend the potential role of this promising MR technique.

As previously stated by Krings et al. ${ }^{11}$, the pathophysiology of thrombosed aneurysms suggests that only surgical trapping may exclude the vasa vasorum connection between the parent artery wall and the aneurysmal neck and ultimately halt the inflammatory changes. However, the role of endovascular flow-diversion devices in treating this subgroup of aneurysms has recently emerged with promising results. ${ }^{17,18}$ By altering hemodynamics at the parent vessel/ aneurysm interface, we hypothesize that in our 2 cases the flow-diverter stents might be responsible for diverting blood flow away from the aneurysm lumen and more importantly from the intrathrombotic blood channels to the vasa vasorum, eventually preventing adventitial inflammation. Further studies may help to expand the treatment options for a partially thrombosed aneurysm presenting with PE.

Our 3 cases suggest a common pathophysiologic mechanism sustaining the occurrence of PE in partially thrombosed aneurysms, regardless of their dimensions and mass effect on brain parenchyma. Thus, intraluminal and intramural thrombus within intracranial aneurysms should be promptly recognized and reported in neuroimaging. In addition, our third case advocates for the possibility that ongoing remodeling and inflammatory processes may exist, even in a fully-thrombosed non-revascularized aneurysm several years after coiling.

\section{Fund}

None.

\section{Ethics Statement}

Our Institutional Review Board (IRB) waived the approval for the present case report.

\section{Conflicts of Interest}

The authors have no conflicts to disclose.

\section{Author Contributions}

Analysis and interpretation: VO. Data collection: VO and EV. Writing the article: $\mathrm{VO}$ and EV. Critical revision of the article: DT, AC, and EV. Final approval of the article: AC and EV. Overall responsibility: $\mathrm{VO}$. 


\section{ORCID}

Valeria Onofrj: https://orcid.org/0000-0003-1170-7282

Donatella Tampieri: https://orcid.org/0000-0002-0329-3262

Alessandro Cianfoni: https://orcid.org/0000-0001-9333-4912

Elisa Ventura: https://orcid.org/0000-0002-9029-6516

\section{REFERENCES}

1. Bose B, Northrup B, Osterholm J. Giant basilar artery aneurysm presenting as a third ventricular tumor. Neurosurgery 1983;13:699-702

2. Heros RC, Kolluri S. Giant intracranial aneurysms presenting with massive cerebral edema. Neurosurgery 1984;15:572-577

3. Craven I, Patel UJ, Gibson A, Coley SC. Symptomatic perianeurysmal edema following bare platinum embolization of a small unruptured cerebral aneurysm. AJNR Am J Neuroradiol 2009;30:1998-2000

4. Lanzino G. Inflammation after embolization of intracranial aneurysms. J Neurosurg 2008;108:1071-1073

5. Lehman VT, Brinjikji W, Kallmes DF, Huston J Rd, Lanzino G, Rabinstein $A A$, et al. Clinical interpretation of high-resolution vessel wall MRI of intracranial arterial diseases. Br J Radiol 2016;89:20160496

6. Shimonaga K, Matsushige T, Ishii D, Sakamoto S, Hosogai M, Kawasumi T, et al. Clinicopathological insights from vessel wall imaging of unruptured intracranial aneurysms. Stroke 2018:49:2516-2519

7. Horie N, Kitagawa N, Morikawa M, Tsutsumi K, Kaminogo M, Nagata I. Progressive perianeurysmal edema induced after endovascular coil embolization. Report of three cases and review of the literature. J Neurosurg 2007;106:916-920

8. lihara K, Murao K, Sakai N, Soeda A, Ishibashi-Ueda H, Yutani $C$, et al. Continued growth of and increased symptoms from a thrombosed giant aneurysm of the vertebral artery after complete endovascular occlusion and trapping: the role of vasa vasorum. Case report. J Neurosurg 2003;98:407-413

9. Krings T, Alvarez H, Reinacher P, Ozanne A, Baccin CE, Gandolfo C, et al. Growth and rupture mechanism of partially thrombosed aneurysms. Interv Neuroradiol 2007;13:117-126

10. Dengler J, Maldaner N, Bijlenga P, Burkhardt JK, Graewe A, Guhl S, et al.; Giant Intracranial Aneurysm Study Group. Perianeurysmal edema in giant intracranial aneurysms in relation to aneurysm location, size, and partial thrombosis. J Neurosurg 2015;123:446452

11. Krings T, Mandell DM, Kiehl TR, Geibprasert S, Tymianski M, Alvarez $\mathrm{H}$, et al. Intracranial aneurysms: from vessel wall pathology to therapeutic approach. Nat Rev Neurol 2011;7:547-559

12. Im SH, Han MH, Kwon BJ, Jung C, Kim JE, Han DH. Aseptic meningitis after embolization of cerebral aneurysms using hydrogel-coated coils: report of three cases. AJNR Am J Neuroradiol 2007;28:511-512

13. Samaniego EA, Roa JA, Hasan D. Vessel wall imaging in intracranial aneurysms. J Neurointerv Surg 2019;11:1105-1112

14. Sexton T, Smyth SS. Novel mediators and biomarkers of thrombosis. J Thromb Thrombolysis 2014;37:1-3

15. Stenmark KR, Yeager ME, El Kasmi KC, Nozik-Grayck E, Gerasimovskaya EV, Li M, et al. The adventitia: essential regulator of vascular wall structure and function. Annu Rev Physiol 2013;75:23-47

16. Larsen N, von der Brelie C, Trick D, Riedel CH, Lindner T, Madjidyar J, et al. Vessel wall enhancement in unruptured intracranial aneurysms: an indicator for higher risk of rupture? High-resolution MR imaging and correlated histologic findings. AJNR Am J Neuroradiol 2018;39:1617-1621

17. Becske T, Kallmes DF, Saatci I, McDougall CG, Szikora I, Lanzino G, et al. Pipeline for uncoilable or failed aneurysms: results from a multicenter clinical trial. Radiology 2013;267:858-868

18. Szikora I, Marosfoi M, Salomváry B, Berentei Z, Gubucz I. Resolution of mass effect and compression symptoms following endoluminal flow diversion for the treatment of intracranial aneurysms. AJNR Am J Neuroradio/ 2013;34:935-939 